\title{
Circuit
}

Musiques contemporaines

\section{Foi et persévérance}

\section{Les 75 ans de François Morel}

\section{Éric Morin}

Volume 12, numéro 1, 2001

Henri Pousseur : visages

URI : https://id.erudit.org/iderudit/902242ar

DOI : https://doi.org/10.7202/902242ar

Aller au sommaire du numéro

Éditeur(s)

Les Presses de l'Université de Montréal

ISSN

1183-1693 (imprimé)

1488-9692 (numérique)

Découvrir la revue

Citer ce document

Morin, É. (2001). Foi et persévérance : les 75 ans de François Morel. Circuit, 12(1), 96-99. https://doi.org/10.7202/902242ar

Ce document est protégé par la loi sur le droit d'auteur. L'utilisation des services d'Érudit (y compris la reproduction) est assujettie à sa politique d'utilisation que vous pouvez consulter en ligne.

https://apropos.erudit.org/fr/usagers/politique-dutilisation/
Cet article est diffusé et préservé par Érudit.

Érudit est un consortium interuniversitaire sans but lucratif composé de l'Université de Montréal, l'Université Laval et l'Université du Québec à Montréal. Il a pour mission la promotion et la valorisation de la recherche. https://www.erudit.org/fr/ 


\title{
Foi et persévérance \\ Les 75 ans de François Morel
}

\author{
Éric Morin
}

L'important, c'est de croire en soi et en son œuvre... et de persévérer!

François Morel.

Même si, pour beaucoup, il est convenu que l'émergence de la musique contemporaine au Québec doit beaucoup à ses pionniers, il n'est pas vain de rappeler aux musiciens et aux mélomanes d'aujourd'hui, de la plus jeune génération surtout, que leur métier et leur passion auraient pu ne jamais éclore, n'eût été de la clairvoyance et du dévouement de leurs prédécesseurs. Puisque malheureusement certains d'entre eux ne sont plus, il est dès lors et plus que jamais de notre devoir de rendre hommage à ceux qui, toujours parmi nous, façonnent encore par leurs créations notre identité artistique et culturelle. Eł quoi de plus approprié qu'un anniversaire!

Né à Montréal en 1926, François Morel célèbre cette année son $75^{\mathrm{e}}$ anniversaire de naissance. Pianiste, compositeur, chef d'orchestre, arrangeur et pédagogue, véritable touche-à-tout, Morel est sans contredit, depuis près de cinquante ans, une des figures significatives du monde musical québécois. Sans son apport précieux, l'histoire de la musique contemporaine québécoise eût été fort différente. 75 ans, 50 ans de carrière, l'histoire d'une vie, l'histoire d'une œuvre...

\section{Une vie}

Initié au piano dès l'âge de 9 ans, Morel entre au Conservatoire de musique de Montréal en 1943 pour y compléter son apprentissage musical. II y fréquente notamment les classes de Claude Champagne (composition). Diplôme en main, le jeune compositeur de 27 ans se démarque déjà, alors que sa pièce Antiphonie est jouée au Carnegie Hall, sous la direction de Leopold Stokowski.

Toutefois, à l'instar d'autres sphères de la société, le public, les institutions et le monde musical canadien français de l'époque, au sein duquel Morel fait ses premières armes, repoussent et découragent le légitime besoin de renouveau de la jeune génération. Pendant que l'Europe musicale émerge des noirceurs de la guerre, supportée par l'utopie postsérielle de la nouvelle avant-garde, la musique canadienne française, dans une attitude que Morel qualifie encore de "provinciale», reste tapie dans le coin sombre du conservatisme. 
Assuré que la musique d'ici doit se mettre sur les rails de la modernité, Morel prend son avenir en main et organise en 1954-1955, conjointement avec Gilles Tremblay et Serge Garant, ce qui se révélera être les deux premiers concerts de musique contemporaine au Québec. Les trois organisateurs y jouent eux-mêmes des œuvres pour piano de Webern, Messiaen et Boulez, ainsi que quelques-unes de leurs propres compositions; Morel y interprète notamment ses Études de sonorité (piano solo, 1954). Les réactions sont vives: on réprouve tout autant que l'on encense. L'ampleur du débat qui en découle vient paver la voie à la formation d'un nouveau groupe qui, en 1956, s'organise autour de Morel et de Garant, auxquels se joignent alors Otto Joachim et Jeanne Landry. Le groupe adopte le nom de "Musique de notre temps"; groupe qui, quelques années plus tard, mènera à la création de la Société de musique contemporaine du Québec (SMCQ).

C'est à la même époque que Morel doit renoncer, contraint par une santé et un financement précaires, à l'«incontournable» séjour culturel en Europe. Cependant, sa soif d'idées nouvelles le mène à rencontrer plusieurs sommités internationales. En 1958, à New York, Edgard Varèse le reçoit chez lui à plusieurs reprises. Varèse a alors 75 ans et est au sommet de son art et de sa gloire. II aura dès lors sur le jeune Canadien français une grande influence, esthétique notamment, qui ne s'est jamais démentie depuis.

Les pressions monétaires et l'appétit pour la praxis pousseront Morel à entreprendre, dès 1956, une collaboration musicale avec la Société Radio-Canada (SRC). Pour cet organisme public, vingt-cing ans durant, il composera et arrangera une quantité importante de musique: indicatifs sonores, thèmes, musiques de séries dramatiques et de le téléthéâtres. Ces années de travail à la pige permettront à Morel d'affiner son métier par la fréquentation assidue d'instrumentistes d'horizons divers (classique, jazz, variétés). II y développera notamment une riche connaissance de l'orchestration et un sens aigu des couleurs orchestrales qu'il mettra à profit dans ses compositions pour le concert.

À l'opposé de certains de ses collègues les plus en vue, Morel se tiendra longtemps loin de la pédagogie. II résume clairement le pourquoi d'un tel parti : "J'ai retardé le moment d'enseigner pour une seule raison, celle de posséder suffisamment d'expérience de vie pour faire profiter les étudiants d'un savoir acquis dans la vraie vie musicale et non en théoricien! " François Morel attendra donc la fin des années 70 avant d'entreprendre une nouvelle carrière, à titre cette fois de professeur à l'École de musique de l'Université Laval. II y enseigne la composition, l'analyse musicale ef l'orchestration pendant près de vingt ans. II y dirige aussi divers ensembles de musique contemporaine. Après l'annonce de sa retraite en 1997, fière de son apport à la communauté universitaire et à la société québécoise, la Faculté de musique le nomme professeur émérite.

\section{Une œuvre}

Son catalogue comprend à ce jour une soixantaine d'œuvres. Leur haut niveau de réalisation artistique sera reconnu dans les grandes villes européennes, en 
Russie, au Japon, en Chine et dans les Amériques, où il est défendu par les chefs les plus réputés: Stokowski, Monteux, Ozawa, Metha, Decker, Markevitch, Abbado, Dutoit.

Même si, fondamentalement, Morel se considère à juste titre comme un compositeur symphonique, suivant l'approche varésienne, sa prédilection va tout d'abord aux instruments à vents. Cédant aux charmes du jazz, Morel privilégie plus particulièrement les cuivres, qu'il manie d'ailleurs avec brio. Conséquemment, le tiers de son catalogue, une vingtaine de compositions, est principalement destiné aux vents, ce qui fait de Morel l'un des compositeurs canadiens les plus prolifiques en ce domaine.

Comme en font foi ses partitions pour ensemble, Morel est à classer parmi les meilleurs orchestrateurs nord-américains. "Mon approche pour composer une œuvre dépend de l'instrumentation à laquelle elle est destinée, car je crois depuis toujours que l'intérêt de la musique réside dans une écriture idiomatique pour les instruments employés", d'affirmer à cet égard le compositeur.

S'il est aisé de reconnaître l'influence de Varèse dans les réalisations de François Morel, nombreux sont les grands compositeurs qui l'ont aidé à se définir : Scriabine, Debussy, Ravel, Stravinsky (période "russe»), Bartók, Schönberg, Messiaen, Boulez, Berio, Carter, Takemitsu ont déjà tous été cités par Morel comme "influence volontaire à l'émergence de sa personnalité artistique.

Aujourd'hui, avec le recul, Morel scinde lui-même l'évolution de son esthétique en trois grandes périodes. Une douzaine d'œuvres, composées de 1946 à 1962, reflètent, dans un langage essentiellement modal, le canevas des recherches du compositeur jusqu'à ce jour : équilibre de la forme dans l'interaction des composantes rythmiques, mélodiques et harmoniques. L'influence des modèles est tout d'abord sensible: Debussy dans Esquisse (orchestre, 1946), Stravinsky dans Diptyque (orchestre à vent, 1948-1954) et Quatuor à cordes $n^{\circ} 1$ 11952), Varèse dans Rituel de l'espace (orchestre, 1958-1959) et Boréal lorchestre, 1959). Les modèles y sont toutefois savamment transcendés, l'expression étant déjà pleinement celle de Morel.

Une seconde grande période (1962-1980), où la série dodécaphonique comme base de la construction sonore sera le fil d'Ariane, se subdivise elle-même en deux phases. Premièrement, dès 1962, Morel ressent le besoin, dans sa quête de l'équilibre formel, de resserrer ses principes d'organisation. Après l'avoir au préalable longuement étudiée, la série lui semble dès lors l'outil le plus efficace pour parvenir à ses fins. Déjà bien au fait du cul-de-sac vers lequel mène une généralisation de la sérialisation à tous les paramètres du sonore, Morel se garde bien d'emprunter cette voie. L'Étoile noire (orchestre, 1962) et Quintette pour cuivres (1962) sont les toutes premières œuvres de Morel à s'échafauder à partir de séries dodécaphoniques. Le Requiem for Winds (1965-1966). Neumes $d^{\prime}$ Espace et Reliefs (1967) et Prismes-Anamorphoses (1967), trois musiques pour 
orchestre à vents, sont à considérer comme les plus significatives de cette phase de l'évolution esthétique de Morel.

Dans un second temps, de 1968 à 1980, notamment avec Départs 117 instruments, 1968-1969), et surtout après des œuvres de conception plus austère telles que Radience (orchestre, 1970) et likkii (18 exécutants, 1971), le compositeur veillera au développement d'un style plus personnel, propice à un certain lyrisme, sans toutefois renoncer à la série dodécaphonique et à la rigueur de l'équilibre formel. Phase de redéfinition esthétique, peu d'œuvres composées alors figurent encore au catalogue de Morel : Me Duele Espana (guitare, 1974-1977) et Jeux (orchestre, 1976) restent les plus citées.

La troisième grande période esthétique de Morel, qui se prolonge jusqu'à aujourd'hui, débute donc au tournant des années 80 , au moment où le compositeur met fin à sa collaboration avec la SRC et entame sa nouvelle carrière de pédagogue. Sûr de son métier, Morel libéralise et métisse ses techniques d'écriture dans l'achèvement d'une stylistique riche et animée. Si une certaine influence de Boulez se fait sentir sur le plan de la formalisation /séries défectives, systèmes de dérivationl, la nouvelle figuration qu'adopte Morel n'est pas sans rappeler certains traits propres à Berio (champs harmoniques fixes, monnayages chatoyants). Morel définit en ces termes cette plus récente avenue : "Mon langage musical est un fait d'amalgame sériel, modal et d'un sentiment tonal dissonant très évolué, soutenu par le miroitement incessant des timbres qui contribuent également à rendre ce sentiment d'espace coloré que je cherche. [...] C'est un monde moderne. Je ne marche pas dans l'espèce de renouveau actuel, le postmodernisme ou les retours en arrière nostalgiques. "

Au cours des années 80, Morel façonne un nombre imposant d'œuvres fortement diversifiées quant à l'instrumentation: Mélisma (piano et orchestre, 1980), L'OiseauDemain 112 flûtes, clarinette basse, clarinette contrebasse et 3 percussions, 19811982), Divergence (violon et guitare, 1983), Lyre de Cristal 16 percussionnistes, 1985-1986), Aux Couleurs du Ciel (orchestre à vents, 1985-1987), Paysage dépaysé (quintette à cordes, 1990). Outre Strophes, Séquences, Mouvements (12 saxophones, 1999), quelques musiques pour cuivres ainsi que des œuvres pour solistes. Notons que cet éclectisme instrumental s'estompe au cours des dix dernières années et les efforts de Morel se concentrent depuis sur la rédaction de pages pour orchestre symphonique dont Die Stelle der Zwillinge (1992), Et le Crépuscule... se trouva libre (1996), Les Récifs du rêves (2000) et Rupture' (2001).

$\grave{A}$ cet égard, parmi les nombreux projets que le compositeur caresse, se trouve celui de composer pour chacun des orchestres symphoniques régionaux du Québec. Écrire de nouveau pour l'Orchestre symphonique de Montréal (OSM) reste aussi l'un des souhaits chers à son cœur.

Fort d'un troisième quart de siècle, créateur maintenant jubilaire, François Morel compose plus que jamais. Pour notre plus égoïste plaisir, souhaitons qu'il en soit ainsi encore longtemps...
1. Rupture a été créée par l'Orchestre symphonique de Québec (OSQ) en mai 2001, lors d'un concert en hommage au compositeur et présenté à guichet fermé. Rigoureusement préparée et admirablement rendue par l'orchestre sous la direction de Jean-François Rivest, cette œuvre confirma une fois de plus au public québécois l'importance du compositeur, de son métier et de son talent. 